\title{
ANÁLISIS DE LOS SEGMENTOS DE ACTUACIÓN DOCENTE EN DIEZ SECUENCIAS DIDÁCTICAS DE PROFESORES DE HISTORIA USANDO TECNOLOGÍAS*
}

\author{
ANALYSES OF TEACHING PERFORMANCE SEGMENTS OF TEN HISTORY \\ TEACHING SEQUENCES WITH THE USE OF TECHNOLOGY
}

\author{
Marcelo Arancibia Herrera**, Carmen Paz Soto Caro ${ }^{* * *}$ y Roberto Casanova Seguel ${ }^{* *}$
}

\begin{abstract}
El presente estudio describe y caracteriza el despliegue didáctico de diez secuencias de clases de Historia en enseñanza secundaria usando tecnologías. El enfoque asumido para el análisis de las Secuencias Didácticas (SD) es la identificación de Segmentos de Actuación Docente (SAD). Los principales resultados muestran la presencia de 13 tipos de uso de tecnologías, cuatro en relación con el uso del profesor y siete usos de los estudiantes. En conclusión, se exhiben SD con poca diversidad en el uso de las tecnologías, esencialmente prácticas asociadas a un enfoque transmisivo de la enseñanza. Los SAD son similares, poco diversos, con variaciones en el despliegue o momento en que las tecnologías son utilizadas por cada profesor.
\end{abstract}

Palabras claves: Didáctica de la historia, uso educativo de tecnologías, prácticas pedagógicas.

The present study describes and characterizes the didactic deployment of ten sequence of history lessons in high school, with the use of technologies. In order to accomplish the analysis the focus was center in the teaching sequence (TS), by the identification of teaching performance segments (TPS). The main results show 13 types of technologic use, four of them associate with the teachers' way of use and seven related to the students. It conclusion, there are many TS with a few diversity; most of them associate to a transmissive approach of teaching. The TPS are very similar, with almost none diversity, the only change is the when they are used by each teacher.

Key words: History didactic, educational use of technologies, pedagogical practices.

\section{Introducción}

La falta de integración de las denominadas ciencias sociales, según Camillioni (1994), es una dificultad para realizar estudios respecto de la enseñanza en el área curricular de Historia, pues este sector curricular es una confluencia de disciplinas que no siempre se trabajan integradas, lo que se traduce en que las concepciones y prácticas de profesores de ciencias sociales pueden variar dependiendo del tema que deben enseñar. Para Armento (2000) resulta muy complicado investigar concerniente a enseñar y aprender Historia en la educación escolar por dos razones fundamentales: la falta de consenso y claridad acerca de los objetivos de los estudios sociales, que trae como consecuencia la falta de acuerdo respecto de los objetivos para la preparación del profesorado en estudios sociales: cada cultura define sus prioridades respecto de lo que se debe transmitir como conocimiento histórico; el nivel general de descontento con el statu quo de la educación en estudios sociales en la secundaria, así como en los programas de formación de profesores, lo que conlleva el bajo interés institucional por realizar investigaciones en esta área específica.

En esta coyuntura el profesor de historia entra en un proceso, tal vez inconsciente, de reflexión y cuestionamiento de su quehacer y de su rol pedagógico y político, lo que configura un marco referencial que marca sus concepciones acerca de enseñar y aprender historia en la escuela. Es así como la historia de la Historia presenta momentos de severas disputas respecto de, por un lado, cómo se ha de realizar el estudio del pasado (cuestiones de orden metodológico), y por otro lado, qué es el pasado y qué importancia tiene estudiarlo (cuestiones de orden epistemológicas) (Braudel 1974; Friera 1995; Pagès 1983). De esta forma se acuña el término conocimiento histórico (Pagès

\footnotetext{
* $\quad$ Resultado del proyecto FONDECYT N 11130316.

** Universidad Austral de Chile, Valdivia, Chile. Correos electrónicos: marceloarancibia@uach.cl; roberto.casanova@uach.cl

*** Universidad San Sebastián, Concepción, Chile. Correo electrónico: carmenpaz.soto@uss.cl
} 
1983), que hace referencia al pasado reconstruido (relatado) mediante los procedimientos propios del historiador.

Hecha esta distinción respecto del objeto de estudio de la historia, emerge una primera cuestión que traerá repercusiones en su enseñanza en contextos escolares: ¿cuál es el contenido de la Historia en las escuelas?, ¿el pasado como lo que ha ocurrido o el pasado que cuenta la historiografía? Pagès (1983:12) presenta esta distinción estableciendo que la historia ha de incluir ambos procesos "El concepto historia incluye, pues, la realidad histórica tal y como objetivamente acaeció, y el conocimiento histórico, o sea la ciencia que pretende develarnos, mediante el trabajo del historiador, la realidad histórica". Más adelante recalca que muchas veces existe una distancia entre estas historias, entre la realidad objetiva y el conocimiento histórico, planteando con ello, en consecuencia, la presencia de múltiples historias.

El planeamiento antes descrito tiene notoria presencia en las discusiones que existen entre las dos corrientes de pensamiento que han primado en el estudio de la Historia (Friera 1995). Por un lado, el positivismo establece que la historia es el relato del pasado que hace la historiografía, lo que se ha de obtener mediante la aplicación rigurosa del método historiográfico con el propósito de eliminar en gran medida la subjetividad, de allí que se construya la imagen del historiador como un ser neutro y su relato como un hecho objetivo. De otro lado se encuentran las posiciones materialistas o revisionistas, donde destaca la Escuela de los Annales de la primera mitad del siglo XX. Esta corriente de pensamiento histórico plantea la presencia de múltiples historias que se multiplican por medio de las interpretaciones que del pasado hacen las personas. Claramente lo manifiesta Braudel (1974:23) cuando plantea que "El espíritu histórico es básicamente crítico. Pero, más allá de prudencias evidentes, es también reconstrucción".

A partir de estas dos visiones sobre la historia es posible deducir implicancias para la didáctica en contextos escolares: por un lado una enseñanza que privilegia la historia de tipo narrativa, perspectiva que podemos denominar como tradicional, en la que el profesor y los libros de texto son los protagonistas; por otro lado una historia crítica o nueva historia (Friera 1995), en donde se privilegia la reconstrucción del pasado local y las visiones revisionistas de la historiografía.

\section{Didáctica de la Historia y Uso de Tecnologías}

No es extraño encontrar que las diferencias descritas subyacen también en las tradiciones epistemológicas de la didáctica de la historia. Según Benejam (1998), esta incidencia conlleva la pregunta ¿para qué enseñamos historia? Esta relación se establece en función de las finalidades de la enseñanza de la historia en la escuela:

La selección de los objetivos generales o finalidades de la educación depende, en gran medida, de la perspectiva teórica en que nos situemos. En efecto, cada manera de entender el mundo prioriza unos principios y conceptos susceptibles de ser aplicados a la enseñanza y establece sus prioridades y valores, de manera que en la epistemología se superponen ciencia e ideología (Benejam 1998:34).

En particular, entrando en el asunto específico de la didáctica de la historia, de acuerdo con Camillioni (1994), esta se entiende como la disciplina que deviene de una teoría respecto de la práctica, en este caso de la práctica de su enseñanza. Por ello, la didáctica no únicamente tiene como campo u objeto de estudio la práctica de la enseñanza de la disciplina, sino también los ámbitos de la reflexión acerca de dicho quehacer:

La reflexión procesa la acción en términos teóricos (...) las propias teorías permiten ir más allá de la simple observación de prácticas propias y ajenas. En la permanente fecundación entre teoría y práctica pedagógica se construye el discurso didáctico (Camillioni 1994:27).

En la didáctica de la historia existen al menos tres ópticas que describen enfoques distintivos respecto de para qué enseñar historia en educación secundaria: instrumental, ciudadano y crítico (Estepa 2000; González 2001; Moradiellos 1994), ellos se describirán a continuación.

a. Enfoque instrumental o técnico. Orientado por una perspectiva que define la historia como una sucesión de acontecimientos entrelazados que deben ser conocidos por los estudiantes, como han sido relatados por la historiografía, independiente de las corrientes historiográficas. Enseñar historia 
sería contar un relato de lo ocurrido, que con posterioridad los estudiantes repiten en un relato con mayor o menor similitud. Este enfoque se encuentra asociado a las corrientes psicopedagógicas de orden conductista y transmisiva.

b. Enfoque ciudadano o práctico. Señala que enseñar historia es formar en los jóvenes conocimientos de orden más bien prácticos, de modo que se configuren como buenos ciudadanos, buscando su participación en la vida cívica de su ciudad. Más que poner énfasis en los relatos historiográficos del pasado, es primordial mirar cómo por medio de la historia se ha conformado nuestro presente, rescatando precisamente el aquí y ahora como elemento fundamental. Desde este enfoque enseñar historia es analizar los procesos históricos como fuente y base del presente de nuestra civilización y cultura.

c. Enfoque emancipatorio o crítico. Centra su quehacer en la perspectiva emancipadora de los sujetos. La historia sería, en este caso, un fenómeno de construcción social, muchas veces escrito de manera sesgada, que no da cuenta de todo el devenir de la humanidad. Enseñar historia es ayudar a descubrir que cada uno puede escribir su propia historia y ayudar a construir una historia colectiva. Por tanto, el centro está puesto en la construcción de un sujeto consciente de su historicidad.

En concordancia con lo anterior, autores como Gimeno $(1998,2002)$ y Kemmis (1998) exhiben la presencia de tres perspectivas curriculares que son homologables con los enfoques antes descritos: un currículo técnico, el que es construido por expertos mediante procesos de estudios y pruebas, que es ejecutado en las aulas sin mediar intervención. Un currículo práctico que, de manera diferente, se sustenta en el hacer emergente, donde poco interesa la reflexión y por tanto se sustenta en acciones automatizadas. Finalmente existe un currículo crítico que pone el foco en la construcción social del conocimiento, por tanto en la reelaboración constante del quehacer educativo. La exposición de los enfoques anteriores denota que no existe una didáctica desinteresada y que no hay una práctica pedagógica neutral. Por ello la acción didáctica, al ser también una praxis social, es el corolario de ciertos procesos teóricos y experiencias que los profesores han ido configurando. De tal forma, asumir uno u otro enfoque no es trivial, pues marca en mayor o menor medida las microsociedades escolares y, por ende, se constituyen en un aporte para la conformación de sociedades más globales.
El recuento de las perspectivas didácticas respecto de cómo y qué enseñar de esta disciplina en la educación secundaria nos ha permitido configurar con bastante claridad una matriz que gira en torno a tres ejes o categorías (Arancibia y Badia, 2013), ellos son los siguientes:

a. La historia es una disciplina que debe enseñar el pasado como un fin en sí mismo, por tanto el estudiante debe reproducir lo que se ha dicho acerca de la historia. Un tipo de práctica asociada a un currículum técnico e instrumental.

b. La historia es un medio para comprender el presente, por tanto el pasado interesa solo como referente global para dar cuenta de por qué las cosas son tal en la actualidad. El aprendizaje, por ello, es también reproductivo, pero contextualizado o significativo. Cercano a un tipo de currículum ciudadano y práctico.

c. La historia es una estrategia para ser autónomos y críticos, principalmente con los modos en que se ha contado la historia, y por ello fomenta el aprendizaje emancipador y se basa en la perspectiva de la cognición distribuida, adscrita a una práctica curricular crítica y emancipadora.

Por otro lado, en el actual contexto educativo parece inevitable vincular los procesos de innovación didáctica al uso de TIC (Ertmer y Ottenbreit-Leftwich 2010; Mueller et al. 2008; Starkey 2010). Ampliando esta perspectiva, la mirada se ha ido diversificando hacia asociar el uso de las TIC con el desarrollo de habilidades o competencias digitales en los estudiantes, apropiadas a los comportamientos y desafíos planteados por la nueva centuria (Gutiérrez y Tyner 2012).

Siguiendo a Ertmer y Ottenbreit-Leftwich (2010), para apoyar el uso eficiente de las TIC hay que atender al menos a cuatro fenómenos: el conocimiento docente de las tecnologías, la autoeficacia de las TIC en sus prácticas, las creencias pedagógicas que construyen los docentes para aprender y enseñar y, por último, la cultura escolar que favorece o dificulta esta incorporación. Con ello, la innovación didáctica con TIC ha de atender a estos cuatro elementos para que se instale, permanezca y provoque aprendizajes.

Por su parte, la escuela no puede seguir reproduciendo prácticas que solo logran aprendizajes superficiales, más bien debe avanzar hacia la obtención de aprendizajes profundos y de calidad, aprendizajes que exige la nueva época digital, orientados hacia habilidades de orden superior, tal 
cual las describe Starkey (2010) en una matriz de seis niveles asociados con prácticas educativas en que se usan TIC: 1) hacer, 2) estableciendo conexiones, 3) pensando en conceptos, 4) crítica y evaluación, 5) creación de conocimiento, 6) compartiendo el conocimiento.

Poniendo en relación las perspectivas epistemológicas con las tradiciones didácticas, configuramos tres enfoques de enseñanza con TIC, ellos se sintetizan en el Cuadro 1 (ver nota 1).

\section{Metodología}

El principal objetivo de este artículo es dar cuenta del despliegue o frecuencia temporal de 10 Secuencias Didácticas (SD) entendidas como las clases de profesores de historia usando tecnologías, con el propósito de identificar diferencias o similitudes en sus prácticas pedagógicas cuando proponen usar tecnología a sus estudiantes. Se trabajó con 6 profesoras y 4 profesores (sin embargo, en el artículo hablaremos indistintamente de profesores sin hacer distinción de género, de modo de privilegiar el anonimato de los participantes), todos se desempeñan en enseñanza media, en colegios de dependencia particular, particular subvencionado y municipal. Los maestros pertenecían a tres regiones del territorio centro sur de nuestro país. Las edades fluctuaron de los 32 a 42 años y los años de ejercicio docente de los 5 a 19 años. Su participación fue voluntaria.

El diseño de la investigación está fundado en casos de tipo múltiple (Stake 1999), busca la comprensión del fenómeno global a partir del estudio de varias realidades. La selección de los casos es de tipo intencionada, se definen criterios a priori que justifica el investigador a partir de particularidades del proceso de indagación. Siguiendo esta lógica, se optó por profesores representativos que den cuenta de la mayor diversidad posible en la enseñanza de la historia con TIC, como se muestra en el Cuadro 2 (ver nota 2).

Para fines de este estudio, la Unidad de Análisis se entiende como el quehacer del profesor durante el desarrollo presencial de una SD en que usa TIC con sus estudiantes, o bien propone el uso por parte de ellos para desarrollar una unidad temática de historia en enseñanza media.

En esta investigación una SD corresponde a la macrounidad de análisis de la práctica docente con uso de TIC, cada profesor desarrolla una SD que ha sido propuesta por él. La característica principal de una SD es que configura un proceso de enseñanza y aprendizaje completo, es decir, que implica en una microescala todos los componentes de este proceso: las etapas de inicio, desarrollo y cierre, el uso de materiales y recursos hasta concluir su uso, diferentes actuaciones de profesores y estudiantes, actividades evaluativas, entre otros. Por otra parte, los Segmentos de Actuación Docente (SAD) se configuran a partir del análisis de las filmaciones de las sesiones de cada profesor, con esto se construye una tipología que define tipos de usos educativos de TIC, en función de lo que hacen los profesores (SADP) y de las actividades que proponen a los estudiantes (SADE). Este análisis de los videos se realiza por medio de los $\mathrm{SAD}$, que se definen como estructuras homogéneas de acción del docente o los estudiantes respecto del uso - $\mathrm{O}$ no uso- que le dan a las TIC durante una SD (Coll et al. 2010).

Para estos hallazgos se analiza de manera cuantitativa y descriptiva la secuencia didáctica mediante diagramas de flujo (Figuras 1-10), mostrándose en tiempo absoluto y orden secuencial los SAD por cada una de las sesiones de las diez SD estudiadas.

Como técnica de validación de los SAD se utiliza la fiabilidad interjueces, calculando el coeficiente Kappa de Cohen (Gros 2008) creado para datos nominales con el objetivo de asegurar la adecuada clasificación realizada por los investigadores de los SAD. Estos fueron sometidos a la revisión externa de dos jueces expertos, lográndose según la escala de Kappa de Cohen, un acuerdo excelente $(>0,75)$ con ambos jueces $(0,86)$.

\section{Resultados}

Los resultados se alcanzan a partir del análisis de las diez SD, las que están constituidas por 2.549 minutos de video, distribuidos en 39 sesiones de clases realizadas a lo largo de este estudio.

A continuación se exponen los SAD identificados del análisis de las SD correspondientes a diez profesores. Como se aprecia en el Cuadro 3, se han diferenciado cuatro SAD en que el profesor usa exclusivamente las TIC, ellos han sido codificados como SADP; mientras en el Cuadro 4 se describen siete SAD donde los estudiantes utilizan las TIC, asignándoles el código SADE. Con esto se observa que son más diversas las actividades en que las TIC son usadas por los estudiantes, en contraste a los segmentos en que las TIC son usadas por los profesores, donde no existe mucha 
Cuadro 1. Caracterización de dimensiones y categorías para aprender y enseñar historia con uso de TIC.

\begin{tabular}{|c|c|c|c|}
\hline \multirow{2}{*}{ Dimensiones } & \multicolumn{3}{|c|}{ Categorías } \\
\hline & Técnico/instrumental & Ciudadano/práctico & Crítico/emancipatorio \\
\hline $\begin{array}{l}\text { Perspectivas teóricas respecto } \\
\text { de la naturaleza del conoci- } \\
\text { miento histórico }\end{array}$ & $\begin{array}{l}\text { El conocimiento histórico es } \\
\text { un hecho objetivo, por tanto su } \\
\text { método ha de ser positivista, } \\
\text { pues requiere ser válido y } \\
\text { confiable }\end{array}$ & $\begin{array}{l}\text { El conocimiento histórico es } \\
\text { un hecho validado por cada } \\
\text { sujeto de acuerdo con lo sig- } \\
\text { nificativo que le resulte, por } \\
\text { tanto su validez es relativa } \\
\text { según cada sujeto }\end{array}$ & $\begin{array}{l}\text { El conocimiento histórico es } \\
\text { una construcción social, por } \\
\text { tanto parte de un discurso } \\
\text { social que lo legitima }\end{array}$ \\
\hline $\begin{array}{l}\text { Concepciones acerca de qué es } \\
\text { enseñar historia en la escuela }\end{array}$ & $\begin{array}{l}\text { Enseñar historia es transmi- } \\
\text { tir los hechos del pasado. } \\
\text { Currículo técnico }\end{array}$ & $\begin{array}{l}\text { Enseñar historia es conseguir } \\
\text { que cada estudiante otor- } \\
\text { gue significado al presente. } \\
\text { Currículo práctico }\end{array}$ & $\begin{array}{l}\text { Enseñar historia es emancipar } \\
\text { el pensamiento. Currículo } \\
\text { crítico }\end{array}$ \\
\hline $\begin{array}{l}\text { Orientaciones para los fines } \\
\text { educativos }\end{array}$ & $\begin{array}{l}\text { La finalidad de la educación } \\
\text { es traspasar el saber acumu- } \\
\text { lado socialmente a las nuevas } \\
\text { generaciones }\end{array}$ & $\begin{array}{l}\text { La finalidad de la educación } \\
\text { es el desarrollo pleno de las } \\
\text { capacidades individuales }\end{array}$ & $\begin{array}{l}\text { El fin de la educación es la } \\
\text { construcción de una sociedad } \\
\text { mejor o más avanzada }\end{array}$ \\
\hline $\begin{array}{l}\text { La importancia concedida al } \\
\text { aprendizaje de historia en la } \\
\text { educación secundaria }\end{array}$ & $\begin{array}{l}\text { Se aprende historia como } \\
\text { contenido valioso en sí mismo }\end{array}$ & $\begin{array}{l}\text { Se aprende historia porque } \\
\text { aporta al desarrollo personal }\end{array}$ & $\begin{array}{l}\text { Se aprende historia para si- } \\
\text { tuarse en un tipo de sociedad }\end{array}$ \\
\hline $\begin{array}{l}\text { Definición de los roles del } \\
\text { profesor }\end{array}$ & $\begin{array}{l}\text { El profesor expone contenidos } \\
\text { disciplinarios y corrige los } \\
\text { errores }\end{array}$ & $\begin{array}{l}\text { El profesor organiza activida- } \\
\text { des y supervisa su desarrollo }\end{array}$ & $\begin{array}{l}\text { El profesor participa de pro- } \\
\text { cesos dialógicos y debates } \\
\text { respecto de la historia }\end{array}$ \\
\hline $\begin{array}{l}\text { Definición de los roles del } \\
\text { estudiante }\end{array}$ & $\begin{array}{l}\text { Reproducir fielmente lo que } \\
\text { se ha enseñado, Conocer el } \\
\text { pasado }\end{array}$ & $\begin{array}{l}\text { Construye sentidos individua- } \\
\text { les de lo que se le ha enseñado. } \\
\text { Interpreta el presente }\end{array}$ & $\begin{array}{l}\text { Toma posicionamiento críti- } \\
\text { co respecto de los procesos } \\
\text { históricos }\end{array}$ \\
\hline $\begin{array}{l}\text { Enfoques o modelos de activi- } \\
\text { dad didáctica }\end{array}$ & $\begin{array}{l}\text { Actividades básicamente de } \\
\text { exposición de contenidos, lec- } \\
\text { tura dirigida, guías de trabajo }\end{array}$ & $\begin{array}{l}\text { Combina actividades de los } \\
\text { otros enfoques, pero con én- } \\
\text { fasis en el trabajo individual }\end{array}$ & $\begin{array}{l}\text { Disertaciones, trabajos de } \\
\text { investigación, debates y } \\
\text { metacognición }\end{array}$ \\
\hline $\begin{array}{l}\text { Importancia asignada a la } \\
\text { planificación de la enseñanza }\end{array}$ & $\begin{array}{l}\text { Referente que sirve al profesor } \\
\text { para ordenar la enseñanza del } \\
\text { año escolar, basándose en el } \\
\text { currículo prescrito }\end{array}$ & $\begin{array}{l}\text { Principalmente un organizador } \\
\text { de actividades que sirve al } \\
\text { profesor para saber lo que } \\
\text { debe hacer clase a clase, para } \\
\text { planificar usa como referente } \\
\text { las características intelectuales } \\
\text { de los estudiantes }\end{array}$ & $\begin{array}{l}\text { No organiza mucho su tra- } \\
\text { bajo, es más bien flexible y } \\
\text { emergente }\end{array}$ \\
\hline $\begin{array}{l}\text { Concepciones para evaluar y } \\
\text { prácticas evaluativas }\end{array}$ & $\begin{array}{l}\text { La evaluación es una rutina es- } \\
\text { tructurada y objetiva, orientada } \\
\text { a los resultados y la calificación }\end{array}$ & $\begin{array}{l}\text { La evaluación es una tarea del } \\
\text { docente que permite retroali- } \\
\text { mentar el aprendizaje de los } \\
\text { estudiantes }\end{array}$ & $\begin{array}{l}\text { La evaluación es un ejerci- } \\
\text { cio colectivo e integral que } \\
\text { permite mejorar las prácticas } \\
\text { pedagógicas }\end{array}$ \\
\hline $\begin{array}{l}\text { Papel asignado a la contextua- } \\
\text { lización del aprendizaje }\end{array}$ & $\begin{array}{l}\text { Contextualiza, principalmente, } \\
\text { en función de ejemplos cerca- } \\
\text { nos a los estudiantes }\end{array}$ & $\begin{array}{l}\text { Usa la contextualización como } \\
\text { dispositivo motivador }\end{array}$ & $\begin{array}{l}\text { Entiende la contextualización } \\
\text { como la construcción de un } \\
\text { espacio compartido (ZDP) }\end{array}$ \\
\hline $\begin{array}{l}\text { Relevancia asignada al uso de } \\
\text { las TIC en la escuela }\end{array}$ & $\begin{array}{l}\text { Fuentes de información que } \\
\text { presentan contenidos }\end{array}$ & $\begin{array}{l}\text { Fuentes de información que } \\
\text { apoyan procesos de profun- } \\
\text { dización e investigación }\end{array}$ & $\begin{array}{l}\text { Medios para encontrar argu- } \\
\text { mentos que están a la base de } \\
\text { las visiones críticas }\end{array}$ \\
\hline $\begin{array}{l}\text { Intencionalidad en el uso de } \\
\text { TIC en la enseñanza de la } \\
\text { Historia }\end{array}$ & $\begin{array}{l}\text { Apoyo a la enseñanza para } \\
\text { exponer información }\end{array}$ & $\begin{array}{l}\text { Apoyo a la enseñanza para } \\
\text { motivar }\end{array}$ & $\begin{array}{l}\text { Aportar a la reflexión y el ma- } \\
\text { nejo crítico de la información }\end{array}$ \\
\hline Rol social de las TIC & $\begin{array}{l}\text { Artefactos neutros propios del } \\
\text { desarrollo humano }\end{array}$ & $\begin{array}{l}\text { Herramientas útiles para hacer } \\
\text { un mejor trabajo }\end{array}$ & Instrumentos alienadores \\
\hline
\end{tabular}


Cuadro 2. Síntesis características socioprofesionales profesores entrevistados.

\begin{tabular}{|c|c|c|c|c|c|}
\hline Profesor & Edad & $\begin{array}{l}\text { Años de } \\
\text { ejercicio }\end{array}$ & Formación profesional & Contexto desempeño & Nivel uso TIC1 \\
\hline P1 & 35 & 8 & Profesora de Historia y Geografía & Subvencionado laico & Avanzado \\
\hline $\mathrm{P} 2$ & 41 & 18 & $\begin{array}{l}\text { Profesora de Historia, Geografía y } \\
\text { Educación cívica }\end{array}$ & Particular & Intermedio \\
\hline P3 & 37 & 10 & $\begin{array}{l}\text { Profesora de Historia, Geografía y } \\
\text { Educación cívica }\end{array}$ & Subvencionado religioso & Avanzado \\
\hline P4 & 32 & 5 & Profesora de Historia y Geografía & Subvencionado laico & Avanzado \\
\hline P5 & 33 & 5 & Profesor de Historia y Geografía & Subvencionado religioso & Intermedio \\
\hline P6 & 38 & 10 & $\begin{array}{l}\text { Profesora de Historia, Geografía y } \\
\text { Educación cívica }\end{array}$ & Subvencionado religioso & Avanzado \\
\hline P7 & 37 & 7 & $\begin{array}{l}\text { Profesor de Historia, Geografía y } \\
\text { Educación cívica }\end{array}$ & Subvencionado laico & Intermedio \\
\hline P8 & 43 & 16 & Profesora de Historia y Geografía & Subvencionado religioso & Intermedio \\
\hline P9 & 42 & 13 & $\begin{array}{l}\text { Profesor de Historia, Geografía y } \\
\text { Educación cívica }\end{array}$ & Subvencionado laico & Avanzado \\
\hline P10 & 42 & 19 & $\begin{array}{l}\text { Profesor de Historia, Geografía y } \\
\text { Educación cívica }\end{array}$ & Subvencionado municipal & Intermedio \\
\hline
\end{tabular}

Cuadro 3. Descripción de categorías SAD con uso de TIC por parte del profesor (SADP).

\begin{tabular}{|c|c|}
\hline Código y nombre SAD & Definición \\
\hline $\begin{array}{l}\text { SADP1 Apoya la exposición } \\
\text { oral }\end{array}$ & $\begin{array}{l}\text { Segmento en donde el profesor expone un contenido con apoyo de algún recurso TIC. La principal } \\
\text { fuente de información es su discurso y el recurso tecnológico es solo un apoyo (visual o textual) a } \\
\text { dicha exposición de contenidos }\end{array}$ \\
\hline $\begin{array}{l}\text { SADP2 Apoya la conversación } \\
\text { con los alumnos }\end{array}$ & $\begin{array}{l}\text { Fragmento de la SD en que el profesor se apoya en algún recurso TIC para generar un diálogo } \\
\text { con los estudiantes, es decir, las TIC son base para generar conversaciones respecto del contenido }\end{array}$ \\
\hline $\begin{array}{l}\text { SADP3 Muestra ejemplos de } \\
\text { productos }\end{array}$ & $\begin{array}{l}\text { Corresponde a los momentos en que las TIC son usadas por el profesor para ejemplificar o modelar } \\
\text { los productos que se espera sean desarrollados por los estudiantes, es decir, en que muestra en pantalla } \\
\text { productos "ideales" respecto de lo solicitado en las tareas a desarrollar durante la secuencia didáctica }\end{array}$ \\
\hline $\begin{array}{l}\text { SADP4 Presenta contenidos } \\
\text { curriculares }\end{array}$ & $\begin{array}{l}\text { Segmentos de la SD en que el contenido está puesto en las TIC. De esta forma, el profesor las usa } \\
\text { para que los estudiantes adquieran la información previamente preparada. En este caso el recurso } \\
\text { TIC contiene y transmite el contenido o información. El propósito del uso de la TIC está puesto } \\
\text { en transmitir información o bien motivar a los estudiantes con algún diaporama que presenta in- } \\
\text { formación temática de la unidad }\end{array}$ \\
\hline
\end{tabular}

variabilidad (análisis más detallado en Arancibia y Badia 2013).

A continuación se exponen los respectivos diagramas seguidos de su análisis.

La Figura 1 muestra que P1 tiene la tendencia a iniciar y cerrar las sesiones sin uso de TIC, así como también presenta largos momentos en que las TIC son utilizadas por los estudiantes durante el desarrollo de las cinco sesiones. Asimismo, es destacable que los estudiantes exponen con apoyo de TIC en dos sesiones ( $3^{\mathrm{a}}$ y $5^{\mathrm{a}}$ sesiones).

En los SAD en que no se usan TIC, el profesor suele generar conversaciones con los estudiantes respecto de los contenidos y actividades desarrollados en la sesión anterior: SAD (1), (4), (8) y (10), o bien puntualiza o recuerda cuestiones de las actividades durante el desarrollo de las sesiones: SAD (2), (5), (9) y (11). En última instancia, al 
Cuadro 4. Descripción de categorías SAD con uso de TIC por parte de los estudiantes (SADE).

\begin{tabular}{|c|c|}
\hline Código y nombre SAD & Definición \\
\hline $\begin{array}{l}\text { SADE1 Acceder } \\
\text { a información } \\
\text { seleccionada }\end{array}$ & $\begin{array}{l}\text { SAD en que los estudiantes, a petición del profesor, se dirigen a buscar un recurso o información específico } \\
\text { que el profesor les indica de manera directa para su revisión }\end{array}$ \\
\hline $\begin{array}{l}\text { SADE2 Apoyar una } \\
\text { disertación }\end{array}$ & $\begin{array}{l}\text { Fragmentos que corresponden al momento en que los estudiantes exponen su trabajo a sus compañeros } \\
\text { apoyados de algún recurso TIC, tipo "disertación". El propósito en este caso es dar cuenta por parte de } \\
\text { los estudiantes de los productos solicitados y del trabajo llevado a cabo durante una sesión, o bien durante } \\
\text { toda la SD }\end{array}$ \\
\hline $\begin{array}{l}\text { SADE3 } \\
\text { Elaborar productos } \\
\text { de aprendizaje }\end{array}$ & $\begin{array}{l}\text { SAD en que los estudiantes deben elaborar un producto o material usando las TIC. Corresponde a una } \\
\text { tarea que les asigna el profesor, cuya ejecución implica hacer algo específico usando alguna TIC, por } \\
\text { ejemplo, una tabla en Word o un blog. El foco aquí no está en la búsqueda de información, sino en que } \\
\text { dicha búsqueda sirva para hacer un producto con ella }\end{array}$ \\
\hline $\begin{array}{l}\text { SADE4 } \\
\text { Realizar ejercicios }\end{array}$ & $\begin{array}{l}\text { Fragmentos en que los estudiantes completan o contestan preguntas. Aquí la TIC cumple un rol evaluativo, } \\
\text { pues en ella está contenido un cuestionario que los estudiantes responden y que servirá al profesor para } \\
\text { evaluar, en alguna medida, los logros. La TIC es un contenedor de respuestas emitidas por los estudiantes } \\
\text { sobre algunos temas de los trabajados }\end{array}$ \\
\hline $\begin{array}{l}\text { SADE5 Visualizar } \\
\text { información }\end{array}$ & $\begin{array}{l}\text { En estos SAD las TIC son usadas por los estudiantes como un recurso para observar algún tipo de material } \\
\text { que el profesor solicita visualizar de manera directa y explícita. El recurso visual expuesto se constituye } \\
\text { en el foco del segmento }\end{array}$ \\
\hline $\begin{array}{l}\text { SADE6 } \\
\text { Seguir instrucciones }\end{array}$ & $\begin{array}{l}\text { Segmentos en que las TIC son usadas para hacer algo muy concreto con un recurso TIC. El profesor indica } \\
\text { los pasos y supervisa que los estudiantes sigan la secuencia hasta llegar al lugar indicado, los estudiantes } \\
\text { manipulan los ordenadores mientras el profesor, oralmente, indica hacia dónde deben dirigirse para llegar } \\
\text { a algún sitio }\end{array}$ \\
\hline $\begin{array}{l}\text { SADE7 Buscar } \\
\text { información }\end{array}$ & $\begin{array}{l}\text { Actividades en que preferentemente los estudiantes buscan información o recursos en Internet sin mayores } \\
\text { restricciones de parte del docente. Corresponde a actividades en que los estudiantes navegan de manera } \\
\text { abierta, buscando información que almacenan y organizan sin mayores indicaciones, el recurso principal } \\
\text { aquí es la web y el proceso se centra en la navegación para encontrar información de los temas requeridos } \\
\text { por el profesor }\end{array}$ \\
\hline
\end{tabular}

final de algunas sesiones solicita apagar los ordenadores para realizar una síntesis de lo realizado: SAD (3), (7) y (12). Es importante mencionar que el SAD (6), donde no se usa TIC, corresponde a una presentación de un grupo de estudiantes que no se apoya en los ordenadores.

La SDP1 de la Figura 1 muestra, en primer lugar, que son los estudiantes quienes de manera preferente usan las TIC, y las usan con persistencia para buscar información, elaborar productos y exponer sus resultados, en tanto el profesor las usa para apoyar conversaciones con los estudiantes.

Como se aprecia del despliegue de la SDP2, el uso de TIC se concentra en tres de las cuatro sesiones, en la sesión 1 casi las TIC no son utilizadas. Asimismo, en las siguientes tres sesiones se observa una gran variabilidad de tipos de uso, en cada una de estas sesiones el profesor expone contenidos (SADP1 y SADP5), también en las sesiones 3 y 4 los estudiantes de manera constante están variando de actividades. Destacan entre las diez SD P6 y P10 como las únicas en que los estudiantes no usan las TIC para exponer.

En cuanto a los SAD en que no se usan las TIC, cabe mencionar que el SAD (1) corresponde a las instrucciones generales del trabajo que se realizará en los ordenadores: el profesor explica lo que se hará en las siguientes cuatro sesiones, corresponde a una delimitación y un llamado de atención. En tanto durante la primera sesión existen tres SAD de no usos de TIC consecutivos: (2), (3) y (4), en los cuales el profesor presenta unos textos que luego se leen en conjunto para por último comentarlos. El profesor suele cerrar las sesiones sin uso de TIC: (6), (8) y (9). En los SAD sin uso de TIC: (5) y (7), el profesor con preferencia da instrucciones, por eso se observan al inicio de la segunda y tercera sesiones.

La SD de la Figura 2 muestra, en primer lugar, que son los estudiantes quienes preferentemente usan las TIC, y las usan en especial para realizar ejercicios a base de información que ha sido antes seleccionada por el profesor, es factible recordar que 


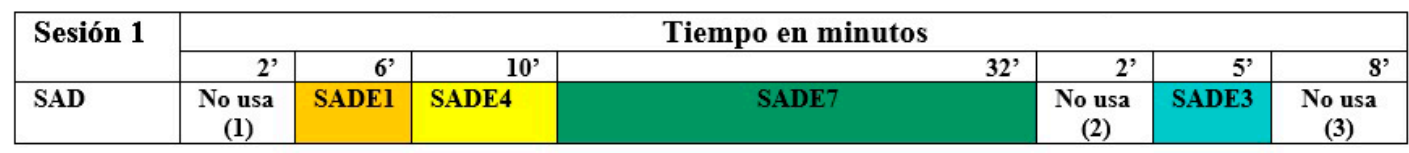

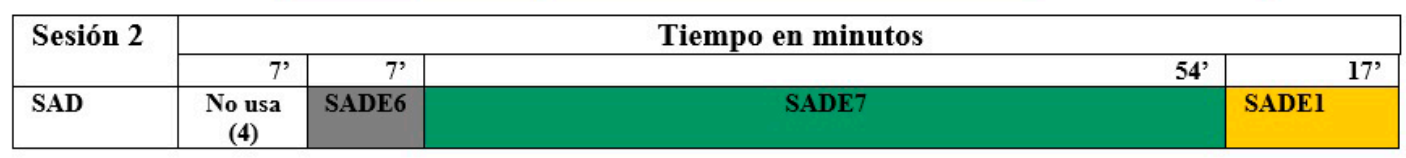

\begin{tabular}{|l|c|cc|r|r|r|r|r|r|}
\hline \multirow{2}{*}{ Sesión 3 } & \multicolumn{8}{|c|}{ Tiempo en minutos } \\
\cline { 2 - 9 } & $5^{\prime}$ & $45^{\prime}$ & $4^{\prime}$ & $2^{\prime}$ & $4^{\prime}$ & $4^{\prime}$ & $3^{\prime}$ & $3^{\prime}$ \\
\hline SAD & SADE6 & SADE3 & & SADE2 & $\begin{array}{c}\text { No usa } \\
(5)\end{array}$ & SADE2 & SADE2 & $\begin{array}{c}\text { No usa } \\
(6)\end{array}$ & $\begin{array}{c}\text { No usa } \\
(7)\end{array}$ \\
\hline
\end{tabular}

\begin{tabular}{|l|r|lr|r|r|r|}
\hline \multirow{2}{*}{ Sesión 4} & \multicolumn{6}{|c|}{ Tiempo en minutos } \\
\cline { 2 - 7 } & $3^{\prime}$ & \multicolumn{6}{|c|}{$\mathbf{2}^{\prime}$} & $2^{2}$ & $3^{\prime}$ \\
\hline SAD & $\begin{array}{c}\text { No usa } \\
\text { (8) }\end{array}$ & SADE3 & $\begin{array}{c}\text { No usa } \\
(9)\end{array}$ & SADP3 & SADP3 \\
\hline
\end{tabular}

\begin{tabular}{|c|c|c|c|c|c|}
\hline \multirow{2}{*}{ Sesión 5} & \multicolumn{5}{|c|}{ Tiempo en minutos } \\
\hline & 2' & 13' & 3' & 27 & 15 ' \\
\hline SAD & $\begin{array}{c}\begin{array}{c}\text { No usa } \\
\text { (10) }\end{array} \\
\text { s. }\end{array}$ & SADE3 & $\begin{array}{l}\text { No usa } \\
\text { (11) }\end{array}$ & $\begin{array}{l}\text { SADP2 } \\
\text { SADE2 }\end{array}$ & $\begin{array}{c}\text { No usa } \\
\text { (12) }\end{array}$ \\
\hline
\end{tabular}

Figura 1. Despliegue de la secuencia didáctica P1.

\begin{tabular}{|c|c|c|c|c|c|c|}
\hline \multirow[t]{2}{*}{ Sesión 1} & \multicolumn{6}{|c|}{ Tiempo en minutos } \\
\hline & $12^{\prime}$ & $4^{\prime}$ & 23 & $14^{\prime}$ & 6' & 4' \\
\hline SAD & $\begin{array}{l}\text { No usa } \\
\text { (1) }\end{array}$ & SADE6 & $\begin{array}{l}\text { No usa } \\
\text { (2) }\end{array}$ & $\begin{array}{l}\text { No usa } \\
\text { (3) }\end{array}$ & $\begin{array}{l}\text { No usa } \\
\text { (4) }\end{array}$ & SADE1 \\
\hline
\end{tabular}

\begin{tabular}{|l|l|l|l|l|l|l|l|l|l|l|}
\hline \multirow{2}{*}{ Sesión 2} & \multicolumn{9}{|c|}{ Tiempo en minutos } \\
\cline { 2 - 10 } & $\mathbf{4}^{\prime}$ & $\mathbf{3}^{\prime}$ & $\mathbf{2 2}^{\prime}$ & $\mathbf{7}^{\prime}$ & $\mathbf{3}^{\prime}$ & $\mathbf{2}^{\prime}$ & $\mathbf{3}^{\prime}$ & $\mathbf{8}^{\prime}$ & $\mathbf{1 0}^{\prime}$ & $\mathbf{2}^{\prime}$ \\
\hline SAD & $\begin{array}{c}\text { No usa } \\
(5)\end{array}$ & SADE6 & SADE4 & SADE4 & SADE1 & SADE4 & SADE3 & SADP1 & $\begin{array}{c}\text { SADE4 } \\
\text { No usa } \\
(6)\end{array}$ \\
\hline
\end{tabular}

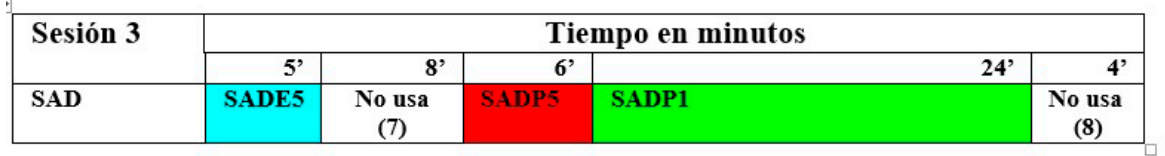

\begin{tabular}{|c|c|c|c|c|c|c|c|}
\hline \multirow[t]{2}{*}{ Sesión 4} & \multicolumn{7}{|c|}{ Tiempo en minutos } \\
\hline & $3^{\prime}$ & $15^{\prime}$ & 2' & $10^{\prime}$ & 15' & 22' & $3^{\prime}$ \\
\hline SAD & SADE5 & SADP1 & SADE6 & SADE1 & SADP1 & SADE4 & $\begin{array}{c}\text { No usa } \\
\text { (9) }\end{array}$ \\
\hline
\end{tabular}

Figura 2. Despliegue de la secuencia didáctica P2.

este profesor es el que más usa las TIC de los diez casos de estudio, no obstante lo hace para exponer o presentar contenidos.

La Figura 3 da cuenta de sesiones reiterativas en el uso de TIC, en las que se realiza la misma acción, salvo en la última donde los estudiantes exponen. $\mathrm{Al}$ respecto, hay que indicar que de los seis grupos que exponen, dos lo hacen sin usar TIC.

De los SAD en que no se usan las TIC, la mayoría son breves instantes en que el profesor recuerda los objetivos y los productos que se han de hacer, lo que ocurre al final de cada sesión: (5), (8), (11), (13) y (15). Asimismo, en los inicios de cada clase recuerda las actividades y contenidos vistos la sesión anterior y entrega las instrucciones de la actividad a desarrollar: (1), (4), (7), (9), (12), (14) y (16). También este profesor comenta, sin hacer uso de las TIC, los recursos con que ha presentado contenido: (2), (3) y (10). Igualmente, durante las disertaciones el profesor aprovecha los 


\begin{tabular}{|c|c|c|c|c|c|c|c|c|}
\hline \multirow[t]{2}{*}{ Sesión 1} & \multicolumn{8}{|c|}{ Tiempo en minutos } \\
\hline & 5' & 3' & $2^{\prime}$ & 5, & 4' & $41^{\prime}$ & 10 ' & 4 \\
\hline SAD & $\begin{array}{l}\text { No usa } \\
\text { (1) }\end{array}$ & SADP4 & $\begin{array}{l}\text { No usa } \\
\text { (2) }\end{array}$ & $\begin{array}{c}\text { No usa } \\
\text { (3) }\end{array}$ & $\begin{array}{c}\text { No usa } \\
\text { (4) }\end{array}$ & SADE7 & $\begin{array}{c}\text { No usa } \\
(5)\end{array}$ & $\begin{array}{l}\text { No usa } \\
\text { (6) }\end{array}$ \\
\hline
\end{tabular}

\begin{tabular}{|l|c|c|c|c|}
\hline \multirow{2}{*}{ Sesión 2 } & \multicolumn{3}{|c|}{ Tiempo en minutos } \\
\cline { 2 - 5 } & \multicolumn{2}{|c|}{ 6' } & \multicolumn{3}{|c|}{ 4' } \\
\hline SAD & $\begin{array}{c}\text { No usa } \\
(7)\end{array}$ & SADEl & $\begin{array}{c}\text { No usa } \\
\text { (s) }\end{array}$ \\
\hline
\end{tabular}

\begin{tabular}{|l|r|r|r|r|r|}
\hline \multirow{2}{*}{ Sesión 3 } & \multicolumn{7}{|c|}{ Tiempo en minutos } \\
\cline { 2 - 6 } & $6^{\prime}$ & $3^{\prime}$ & l' $^{\prime}$ & 2' \\
\hline SAD & $\begin{array}{c}\text { No usa } \\
(9)\end{array}$ & SADP4 & $\begin{array}{c}\text { No usa } \\
(10)\end{array}$ & SADE7 & $\begin{array}{c}\text { No usa } \\
\text { (11) }\end{array}$ \\
\hline
\end{tabular}

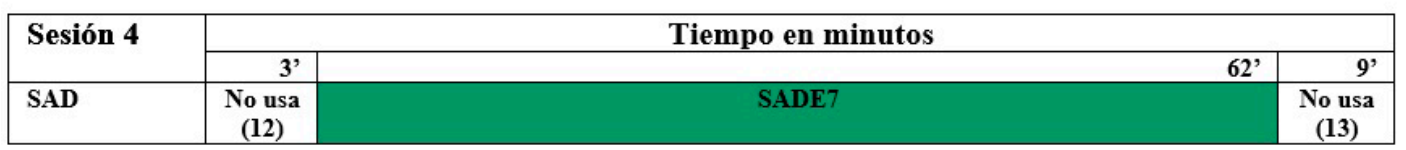

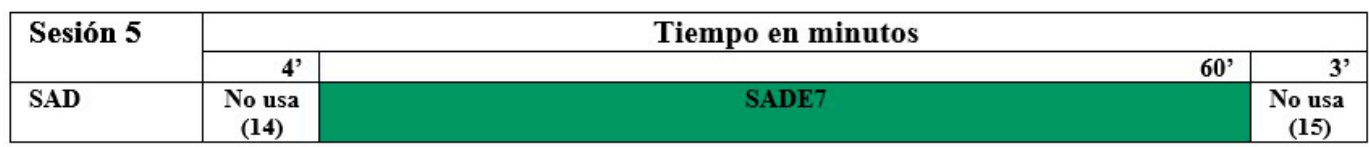

\begin{tabular}{|c|c|c|c|c|c|c|c|c|}
\hline \multirow[t]{2}{*}{ Sesión 6} & \multicolumn{8}{|c|}{ Tiempo en minutos } \\
\hline & 2', & 2 & 4 ' & 5 ' & 4 ' & 10 ' & 5 , & 4' \\
\hline SAD & $\begin{array}{c}\text { No usa } \\
\text { (16) }\end{array}$ & SADE2 & SADE2 & $\begin{array}{c}\text { No usa } \\
\text { (17) }\end{array}$ & SADE2 & SADE2 & $\begin{array}{c}\text { No usa } \\
\text { (18) }\end{array}$ & $\begin{array}{c}\text { No usa } \\
\text { (19) }\end{array}$ \\
\hline
\end{tabular}

Figura 3. Despliegue de la secuencia didáctica P3.

instantes entre una y otra exposición para reforzar los contenidos: (17), (18). Finalmente, P3 cierra la SD sin usar las TIC (19).

La SD de P3 que se ha expuesto en la Figura 3 es una SD en donde los estudiantes son quienes de forma preferente usan las TIC, y las utilizan principalmente para buscar información y exponer con apoyo de ellas, constituyéndose este como el caso en que menos usa las TIC el profesor.

Observamos en la Figura 4 que P4 propone exposiciones de los estudiantes con uso de TIC en la segunda sesión, se dica casi toda la clase a esta actividad. En tanto las sesiones 1 y 3 son de formato prácticamente idéntico, con un primer momento de exposición del profesor, para luego pasar a un largo segmento en que los estudiantes buscan información y documentos.

En cuanto a los SAD en que no se usan las TIC, observamos que existen estos momentos al inicio y al final en cada sesión. Al inicio de las sesiones P4 define los objetivos y contenidos generales sin usar las TIC: (1) y (6), así también organiza las exposiciones (4). En tanto el final de cada sesión corresponde a momentos para preparar el cierre de la clase y adelantar lo que se hará en la siguiente sesión: (5) y (8). Solo en el SAD (3) de no uso de TIC el profesor desarrolla contenidos de modo oral. En tanto los SAD (2) y (7) son básicamente de instrucción para realizar el trabajo de la respectiva sesión tras su exposición oral de contenidos.

La SD de P4 exhibida en la Figura 4 muestra también que son los estudiantes quienes de modo preferente usan las TIC, y las usan principalmente para realizar búsqueda de información y documentos y para exponer, mientras el profesor solo se apoya en ellas para exponer contenidos de forma oral.

Como se aprecia la Figura 5, este profesor concentra su exposición de contenidos en la primera sesión de la SD, al finalizar esta exposición dirige a los estudiantes hacia el blog donde trabajarán para extraer información. La segunda y tercera sesiones son idénticas, un SAD al inicio en que no usan TIC seguido de actividades en que los estudiantes buscan información y documentos hasta el cierre de la clase. En la cuarta sesión los grupos exponen los resultados de los trabajos finales de la SD.

Esta SD presenta la menor presencia de SAD en que no se usan TIC (junto con P10). De estos cuatro $\mathrm{SAD}$, dos corresponden a lectura de noticias de contingencia por parte de los estudiantes 


\begin{tabular}{|c|c|c|c|c|c|c|}
\hline \multirow[t]{2}{*}{ Sesión 1} & \multicolumn{6}{|c|}{ Tiempo en minutos } \\
\hline & 4' & 9' & ', & $43^{\prime}$ & 6' & 7 \\
\hline SAD & $\begin{array}{c}\text { No usa } \\
\text { (1) }\end{array}$ & SADP1 & $\begin{array}{l}\text { No usa } \\
\text { (2) }\end{array}$ & SADE7 & SADE5 & $\begin{array}{l}\text { No usa } \\
\text { (3) }\end{array}$ \\
\hline
\end{tabular}

\begin{tabular}{|c|c|c|c|c|c|c|c|}
\hline \multirow[t]{2}{*}{ Sesión 2} & \multicolumn{7}{|c|}{ Tiempo en minutos } \\
\hline & $5^{\prime}$ & 5 & 7 ' & $6^{\prime}$ & $6^{\prime}$ & 5 & 6, \\
\hline SAD & $\begin{array}{l}\text { No usa } \\
\text { (4) }\end{array}$ & SADE2 & SADE2 & SADE2 & SADE2 & SADE2 & $\begin{array}{c}\text { No usa } \\
\text { (5) }\end{array}$ \\
\hline
\end{tabular}

\begin{tabular}{|c|c|c|c|c|c|}
\hline \multirow[t]{2}{*}{ Sesión 3} & \multicolumn{5}{|c|}{ Tiempo en minutos } \\
\hline & 2' & 3' & 3' & 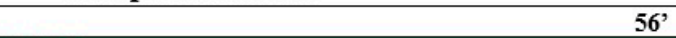 & $2^{\prime}$ \\
\hline SAD & $\begin{array}{c}\text { No usa } \\
\text { (6) }\end{array}$ & SADPl & $\begin{array}{l}\text { No usa } \\
\text { (7) }\end{array}$ & SADE7 & $\begin{array}{c}\text { No usa } \\
\text { (8) }\end{array}$ \\
\hline
\end{tabular}

Figura 4. Despliegue de la secuencia didáctica P4.

\begin{tabular}{|c|c|c|c|c|c|}
\hline \multirow[t]{2}{*}{ Sesión 1} & \multicolumn{5}{|c|}{ Tiempo en minutos } \\
\hline & 2' & 5 & $3^{\prime}$ & $55^{\prime}$ & 15' \\
\hline SAD & SADP1 & SADE5 & SADP1 & SADP1 & SADE1 \\
\hline
\end{tabular}

\begin{tabular}{|c|c|c|c|}
\hline \multirow[t]{2}{*}{ Sesión 2} & \multicolumn{3}{|r|}{ Tiempo en minutos } \\
\hline & 5 ' & 2' & $45^{\prime}$ \\
\hline SAD & $\begin{array}{c}\text { No usa } \\
\text { (1) }\end{array}$ & $\begin{array}{l}\text { No usa } \\
\text { (2) }\end{array}$ & SADE7 \\
\hline
\end{tabular}

\begin{tabular}{|c|c|c|c|}
\hline \multirow[t]{2}{*}{ Sesión 3} & \multicolumn{3}{|r|}{ Tiempo en minutos } \\
\hline & 7 ' & $4 '$ & $61^{\prime}$ \\
\hline SAD & $\begin{array}{c}\text { No usa } \\
\text { (3) }\end{array}$ & $\begin{array}{c}\text { No usa } \\
\text { (4) }\end{array}$ & SADE7 \\
\hline
\end{tabular}

\begin{tabular}{|l|ll|l|l|l|l|}
\hline \multirow{2}{*}{ Sesión 4 } & \multicolumn{7}{|c|}{ Tiempo en minutos } \\
\cline { 2 - 6 } & \multicolumn{9}{|c|}{$18^{\prime}$} & $10^{\prime}$ & \multicolumn{3}{c|}{ 13' } & 11' \\
\hline SAD & SADE2 & & SADE2 & SADE2 & SADE2 \\
\hline
\end{tabular}

Figura 5. Despliegue de la secuencia didáctica P5.

que traen preparadas desde sus casas: (1) y (3), y que no tienen que ver con las actividades ni con el contenido propuesto en la SD. En los SAD (2) y (3) en que no se usan TIC, el profesor entrega las instrucciones del trabajo que se desarrollará durante la sesión.

La Figura 5 muestra que en la SD de P5 también son los estudiantes quienes preferentemente usan las TIC, y las usan principalmente para realizar búsqueda de información y documentos y apoyar sus exposiciones, en tanto el profesor las usa solo para exponer contenidos.

La Figura 6 muestra que este profesor tiene la tendencia a iniciar y cerrar las sesiones sin uso de TIC, así como también presenta largos momentos en que las TIC son utilizadas por los estudiantes durante el desarrollo de las sesiones. Asimismo, llama la atención que los estudiantes no expongan los productos que elaboran en la cuarta sesión.
La SD de P6 expuesta en la Figura 6 nos muestra que son los estudiantes quienes preferentemente usan las TIC, y las usan preponderantemente para buscar información y elaborar productos, en tanto el profesor las usa de modo muy marginal y solo para exponer contenidos en la primera sesión.

Como se aprecia del despliegue de la SD de P7 en la Figura 7, el uso de TIC se concentra en el trabajo de los estudiantes, ya sea elaborando un producto de aprendizaje (SADE3) o realizando ejercicios (SADE8). Estos últimos corresponden a dos segmentos: en el primero interviene un mapa conceptual que el profesor exhibe en los computadores, en el segundo construyen gráficos en planilla Excel según datos extraídos de Internet. En cuanto al segmento SADE3, los estudiantes escriben un documento en Word según el contenido que el profesor presenta en el segmento anterior sin uso de TIC (5). 


\begin{tabular}{|l|r|r|r|c|}
\hline \multirow{2}{*}{ Sesión 1 } & \multicolumn{5}{|c|}{ Tiempo en minutos } \\
\cline { 2 - 6 } & $4^{\prime}$ & $6^{\prime}$ & 29' & $7^{\prime}$ \\
\hline SAD & $\begin{array}{c}\text { No usa } \\
\text { (1) }\end{array}$ & SADP1 & SADE7 & $\begin{array}{c}\text { No usa } \\
\text { (2) }\end{array}$ \\
\hline
\end{tabular}

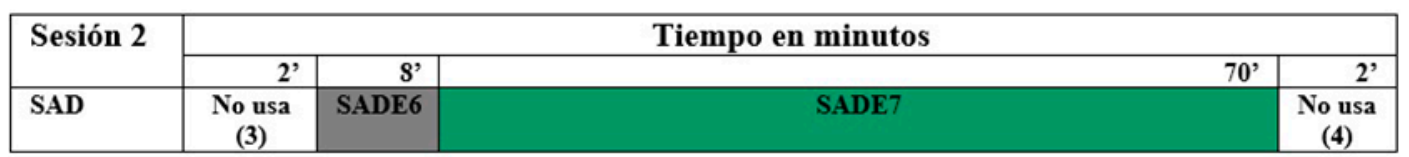

\begin{tabular}{|l|c|cr|r|}
\hline \multirow{2}{*}{ Sesión 3 } & \multicolumn{3}{|c|}{ Tiempo en minutos } \\
\cline { 2 - 5 } & $3^{\prime}$ & \multicolumn{2}{c|}{ 15' $^{\prime}$} \\
\hline SAD & $\begin{array}{c}\text { No usa } \\
\text { (4) }\end{array}$ & SADE7 & SADE4 \\
\hline
\end{tabular}

\begin{tabular}{|l|r|cc|c|}
\hline \multirow{2}{*}{ Sesión 4 } & \multicolumn{4}{|c|}{ Tiempo en minutos } \\
\cline { 2 - 5 } & $3^{\prime}$ & & 79 & $4^{\prime}$ \\
\hline SAD & $\begin{array}{c}\text { No usa } \\
(5)\end{array}$ & SADE3 & $\begin{array}{c}\text { No usa } \\
\text { (6) }\end{array}$ \\
\hline
\end{tabular}

Figura 6. Despliegue de la secuencia didáctica P6.

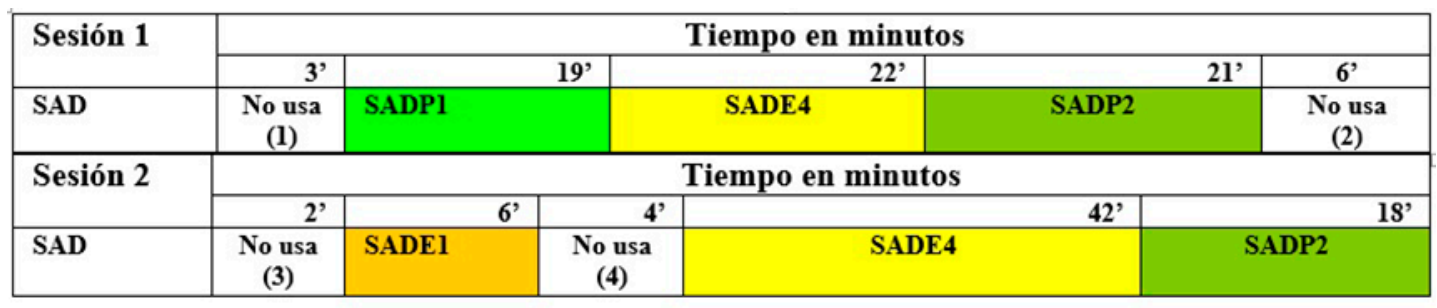

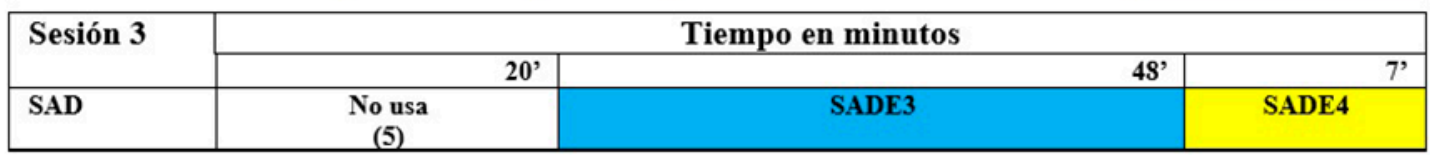

Figura 7. Despliegue de la secuencia didáctica P7.

Por su parte, P7 en la sesión 1 usa las TIC de dos modos: en primer lugar para apoyar su exposición oral (mapa conceptual) y luego al cierre para apoyar la conversación con los estudiantes por medio de la intervención del mapa conceptual y apoyándose en los trabajos realizados por los estudiantes en el segmento anterior.

En la sesión 2 el uso de las TIC de parte del profesor es igualmente para apoyar la conversación con los estudiantes, pues lo hace en función de los gráficos que los estudiantes han elaborado en sus ordenadores. Los momentos en que no se usa TIC son básicamente para organizar la sesión y el trabajo: (1) y (3), así como el cierre en una de ellas (2).

En síntesis, la SD de P7 expuesta en la Figura 7 muestra que son los estudiantes quienes preferentemente usan las TIC para realizar ejercicios sobre la base de información que ha sido previamente seleccionada por el profesor. Resulta interesante recordar que este profesor presenta un alto porcentaje de uso de las TIC, pero como vemos es mayoritariamente para apoyar las conversaciones con los estudiantes.

La Figura 8 da cuenta de una secuencia en que los estudiantes trabajan en la elaboración de productos de aprendizaje durante las dos primeras sesiones, para luego exponerlos a sus compañeros. Es esencial también hacer notar que en todas las sesiones de esta SD el profesor inicia y cierra sin usar las TIC. La SD de P8 es la SD en donde son los estudiantes quienes preferentemente usan las TIC, y las utilizan principalmente para elaborar productos y exponer. 


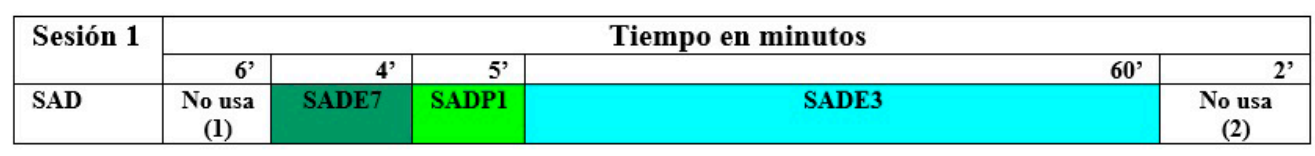

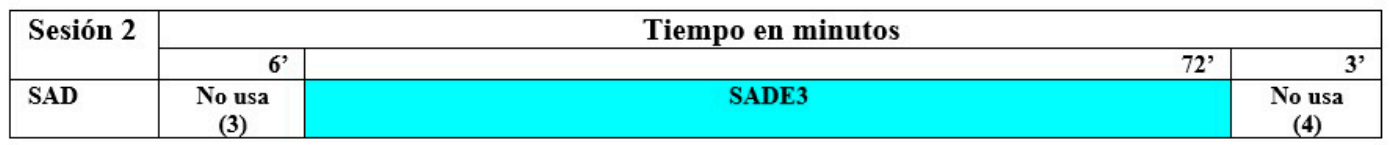

\begin{tabular}{|c|c|c|c|c|c|c|c|c|c|c|c|}
\hline \multirow{2}{*}{ Sesión 3} & \multicolumn{11}{|c|}{ Tiempo en minutos } \\
\hline & $8^{\prime}$ & $7^{\prime}$ & $6^{\prime}$ & 6' & 8, & 9' & 8, & $8^{\prime}$ & $9^{\prime}$ & $6^{\prime}$ & $3^{\prime}$ \\
\hline SAD & $\begin{array}{l}\text { No usa } \\
\text { (5) }\end{array}$ & SADE2 & SADE2 & SADE2 & SADE2 & SADE2 & SADE2 & SADE2 & SADE2 & SADE2 & $\begin{array}{c}\text { No usa } \\
\text { (5) }\end{array}$ \\
\hline
\end{tabular}

Figura 8. Despliegue de la secuencia didáctica P8.

\begin{tabular}{|c|c|c|c|c|c|}
\hline \multirow{2}{*}{ Sesión 1} & \multicolumn{5}{|c|}{ Tiempo en minutos } \\
\hline & 6 ' & 3' & 5, & 38' & $8^{\prime}$ \\
\hline SAD & $\begin{array}{c}\text { No usa } \\
\text { (1) }\end{array}$ & SADP3 & $\begin{array}{c}\text { No usa } \\
\text { (2) }\end{array}$ & SADE7 & SADE4 \\
\hline \multirow[t]{2}{*}{ Sesión 2} & \multicolumn{5}{|c|}{ Tiempo en minutos } \\
\hline & 10 & 5, & 3' & $37^{\prime}$ & $15^{\prime}$ \\
\hline SAD & $\begin{array}{l}\text { No usa } \\
\text { (3) }\end{array}$ & $\begin{array}{c}\text { No usa } \\
\text { (4) }\end{array}$ & $\begin{array}{c}\text { No usa } \\
\text { (5) }\end{array}$ & SADP1 & SADE4 \\
\hline
\end{tabular}

\begin{tabular}{|l|c|}
\hline \multirow{2}{*}{ Sesión 3 } & Tiempo en minutos \\
\cline { 2 - 3 } & 75 \\
\hline SAD & SADE3 \\
\hline
\end{tabular}

\begin{tabular}{|c|c|c|c|c|c|c|c|}
\hline \multirow[t]{2}{*}{ Sesión 4} & \multicolumn{7}{|c|}{ Tiempo en minutos } \\
\hline & $5^{\prime}$ & $5^{\prime}$ & 5 & $7^{\prime}$ & $5^{\prime}$ & 6' & $12^{\prime}$ \\
\hline SAD & SADE4 & SADE2 & SADE2 & SADE2 & SADE2 & SADE2 & $\begin{array}{r}\text { No usa } \\
\text { (5) }\end{array}$ \\
\hline
\end{tabular}

Figura 9. Despliegue de la secuencia didáctica P9.

Observamos en la Figura 9 que $\mathrm{P} 9$ propone una secuencia en que los estudiantes realizan lo siguiente: primero buscan información, luego elaboran un producto (diaporama) que en la última sesión exponen. En la segunda sesión el profesor expone a los estudiantes el uso del programa informático que será empleado para construir el diaporama (Movie maker), el que es luego ejercitado por los estudiantes en el segmento siguiente. En esta SD llama la atención la sesión 3 , en donde los estudiantes trabajan sin interrupciones, elaborando el producto de aprendizaje que luego expondrán.

La SD de P9 muestra también que son los estudiantes quienes de modo preferente usan las TIC, principalmente para elaborar el producto que luego exponen en la última sesión, mientras que el profesor se apoya preferentemente en ellas para exponer oralmente información.

Como se aprecia de la Figura 10, P10 concentra su exposición de contenidos en la primera sesión de la $\mathrm{SD}$, en las siguientes sesiones son los estudiantes quienes realizan un trabajo basado en la búsqueda de información. Es conveniente notar que en varios segmentos P10 está dando instrucciones en relación con el ingreso a los documentos, páginas web y producto que deben realizar, proyectando en la pizarra la secuencia.

La Figura 10 muestra que en la SDP10 también son los estudiantes quienes preferentemente usan las TIC, principalmente para elaborar un producto 


\begin{tabular}{|c|c|c|c|c|c|c|c|}
\hline \multirow[t]{2}{*}{ Sesión 1} & \multicolumn{7}{|c|}{ Tiempo en minutos } \\
\hline & 2' & 3' & 9' & 11 ' & $10^{\prime}$ & $32^{\prime}$ & 3 , \\
\hline SAD & $\begin{array}{l}\text { No usa } \\
\text { (1) }\end{array}$ & SADE6 & SADEl & SADP1 & SADE6 & SADP1 & $\begin{array}{l}\text { No usa } \\
\text { (2) }\end{array}$ \\
\hline
\end{tabular}

\begin{tabular}{|l|r|r|r|r|r|r|}
\hline \multirow{2}{*}{ Sesión 2 } & \multicolumn{6}{|c|}{ Tiempo en minutos } \\
\cline { 2 - 7 } & \multicolumn{2}{|c|}{$\mathbf{2}^{\prime}$} & $\mathbf{6}^{\prime}$ & \multicolumn{2}{|c|}{ 9' $^{\prime}$} & \multicolumn{2}{|c|}{ 15' } & 33' \\
\hline SAD & $\begin{array}{c}\text { No usa } \\
\text { (3) }\end{array}$ & SADE6 & SADE1 & SADP3 & SADE7 \\
\hline
\end{tabular}

\begin{tabular}{|c|c|c|c|c|}
\hline \multirow[t]{2}{*}{ Sesión 3} & \multicolumn{4}{|c|}{ Tiempo en minutos } \\
\hline & 5 , & 2' & $54^{\prime}$ & 12' \\
\hline SAD & $\begin{array}{c}\text { No usa } \\
\text { (4) }\end{array}$ & SADE6 & SADE3 & SADE6 \\
\hline
\end{tabular}

Figura 10. Despliegue de la secuencia didáctica P10.

y realizar búsqueda de información y documentos, en tanto el profesor las usa para exponer contenidos.

\section{Conclusiones}

El despliegue y distribución de los SAD en los diez profesores permite sostener que los docentes del estudio usan las TIC preferentemente para exponer contenidos, fundamentalmente con el propósito de apoyar su exposición oral (salvo P1), y en menor medida para mostrar ejemplos (SADP3) o apoyar la conversación con los estudiantes (SADP2). Los estudiantes son quienes en la mayoría de los casos hacen usos de TIC, fundamentalmente para buscar información en Internet (SADE7), acceder a información previamente definida por el profesor (SADE1) o elaborar productos de aprendizaje (SADE3). También es frecuente el uso de las TIC asociado a exposiciones de los estudiantes (SADE2).

Los resultados del análisis de los usos educativos de las TIC muestran la baja presencia de SADP en las SD y un uso de TIC preferentemente instrumental. Existe poca variedad de usos de TIC cuando estas son empleadas por el profesor. Por otra parte, hay mayor variedad en las propuestas que el profesor hace para el uso de TIC de parte de los estudiantes, sin embargo las SD estudiadas son didácticamente poco variadas (Sigales et al. 2009).

Como síntesis de lo anterior, lo habitual en las SD analizadas fue hallar prácticas con una baja presencia de tipos de uso constructivistas, con ello no se confirma lo mencionado por Hermans et al. (2008), Judson (2006) o Matzen y Edmus (2007) respecto de que las TIC favorecerían la presencia de ese tipo de prácticas.
Por otro lado, si bien existe mayor variabilidad de los tipos de SADE centrados en el uso de los alumnos, lo que indica que cuando los profesores del estudio piensan en el uso de TIC lo hacen en función de la utilización de los estudiantes (SADE), sin embargo, este uso no logra desarrollar altos niveles de aprendizaje (Starkey 2010).

Por tanto, al igual que estudios recientes, los resultados muestran un uso instrumental de las TIC, el que obedece en parte a la falta de diversidad u oferta didáctica del profesor hacia sus estudiantes (Nussbaum et al. 2012). Ante esto, cualquier iniciativa que se proponga debe considerar el trabajo mancomunado de todos los actores del proceso educativo (Rawatlal 2012). Al parecer, se requiere de una innovación integral arraigada tanto en la formación de profesores como en la identidad del establecimiento (Levin y Wadmany 2006; Matzen y Edmunds 2007).

Estos resultados exigen seguir profundizando en los usos reales de las TIC (Coll 2010), es fundamental realizar investigaciones en las aulas donde se usa tecnología, es decir, observar secuencias didácticas naturales que permitan ahondar en el análisis de las prácticas, de modo que los profesores transformen sus prácticas analizando sus propias experiencias y la de otros.

Finalmente, nuestra investigación muestra que, si bien los profesores otorgan mayor protagonismo a los estudiantes en el uso de las TIC, estas actividades no son constructivistas, ello se debe en parte a que sus estructuras didácticas siguen ancladas en concepciones pedagógicas tradicionales (Arancibia y Badia 2015), lo que corrobora que la incorporación de TIC como artefactos que 
sirvan a la instalación de procesos innovadores y que promuevan la renovación de las prácticas no ha tenido el éxito pronosticado en las escuelas. Las TIC en sí mismas no son artefactos que cambien las prácticas, se debe dar en el sistema escolar un paso más allá del simple acto de instalar ordenadores o de promover por decreto la innovación.

\section{Agradecimientos}

A Conicyt por el financiamiento del proyecto Fondecyt Iniciación $\mathrm{N}^{\circ} 11130316$ y al auspicio de la Dirección de Investigación y Desarrollo de la Universidad Austral de Chile.

\section{Referencias Citadas}

Arancibia, M. y Badia, A.

2015 Concepciones de profesores de secundaria sobre enseñar y aprender historia con TIC. Revista Electrónica de Investigación Educativa 17(2):62-76. Recuperado desde http://redie.uabc.mx/vol17no2/contenido-arancibia.html (20 febrero 2016).

Arancibia, M. y Badia, A.

2013 Caracterización y valoración de los usos educativos de las TIC en 10 secuencias didácticas de historia en enseñanza secundaria. Estudios Pedagógicos 39 Número Especial:7-24.

Armento, B.

2000 El desarrollo profesional de los profesores de ciencias sociales. En Modelos, Contenidos y Experiencias en la Formación del Profesorado de Ciencias Sociales, editado por J. Pagès, pp. 19-39. Publicaciones de la Universidad de Huelva, Huelva.

Benejam, P.

1998 Las finalidades de la educación social. En Enseñar y Aprender Ciencias Sociales, Geografía e Historia en la Educación Secundaria, coordinado por P. Benejam y J. Pagés, pp. 33-51. Horsi, Barcelona.

Braudel, F.

1974 La Historia y las Ciencias Sociales. Alianza, Madrid.

Camillioni, A.

1994 Epistemología de la didáctica de las ciencias sociales. En: Didáctica de las Ciencias Sociales. Aportes y Reflexiones, editado por B. Aisenberg y S. Alderoqui, pp. 11-25. Paidos, Buenos Aires.

Coll, C.; Rochera, M. y Colomina, R.

2010 Situated uses of ICT and mediation of Joint activity in a primary education instructional sequence. Electronic Journal of Research in Educational Psychology, 8, 2, 517-540.

Ertmer, P. y Ottenbreit-Leftwich, A.

2010 Teacher Technology Change: How Knowledge, Confidence, Beliefs, and Culture Intersect. Journal of Research on Technology in Education 42(3):255-284.

Estepa, J.

2000 La investigación sobre el conocimiento profesional de los Profesores para enseñar ciencias sociales. Cuadernos de Investigación Didáctica en las Ciencias Sociales 4. Recuperado desde: http://goo.gl/56Jt4 (20 febrero 2016).

Friera, F.

1995 Didáctica de las ciencias sociales. Geografía e Historia. Ediciones de la Torre, Madrid.

Gimeno, J.

1998 Didáctica y Currículo. Morata, Barcelona.

Gimeno, J.

2002 El Curriculum una Reflexión Sobre la Práctica. Morata, Madrid.
González, I.

2001 El juego en la historia social y el juego en el aprendizaje de las ciencias sociales. Iber. Didáctica de las Ciencias Sociales 30:7-22.

Gros, B.

2008 Aprendizajes, Conexiones y Artefactos. La Producción Colaborativa del Conocimiento. Gedisa, Barcelona.

Gutiérrez, A. y Tyner, K.

2012 Educación para los medios, alfabetización mediática y competencia digital. Comunicar 38(XIX):31-39.

Hermans, R.; Tondeur, J.; Van Braak, J. y Valcke, M.

2008 The impact of primary school teachers' educational beliefs on the classroom use of computers. Computers \& Education 51:1499-1509.

Judson, E.

2006 How Teachers Integrate Technology and Their Beliefs About Learning: Is There a Connection? Technology and Teacher Education 14(3):581-597.

Kemmis, S.

1998 El Curriculum: Más Allá de la Teoría de la Reproducción. Morata, Madrid.

Levin, T. y Wadmany, R.

2006 Teachers' Beliefs and Practices in Technology-based Classrooms: A Developmental View. Journal of Research on Technology in Education 39(2):157-181.

Matzen, N. y Edmunds, J.

2007 Technology as a Catalyst for Change: The Role of Professional Development. Journal of Research on Technology in Education 39(4):417-430.

Moradiellos, E.

1994 El Oficio de Historiador. Siglo XXI, Madrid.

Mueller, J.; Wooda, E.; Willoughby, T.; Ross, C. y Specht, J.

2008 Identifying discriminating variables between teachers who fully integrate computers and teachers with limited integration. Computers \& Education 51:1523-1537.

Nussbaum, M.; Weitz, J.; Sibils, X.; Díaz, A. y Claro, M.

2012 Evaluación de la Implementación de la Estrategia Laboratorios Móviles Computacionales (LMC). Informe Final Proyecto Fonide $n^{\circ} 511051$. Recuperado desde http:// centroestudios.mineduc.cl (20 febrero 2016).

Pagès, $\mathrm{P}$.

1983 Introducción a la Historia. Epistemología, Teoría y Problema de Método en los Estudios Históricos. Barcanova, Barcelona.

Rawatlal, K.

2012 Factors impeding school connectedness: a case study South African. Journal of Psychology 42(3):346-357. 
Sigalès, C.; Mominó, J.; Meneses, J. y Badia, A.

2009 La Integración de Internet en la Educación Escolar Española. Ariel, Barcelona.

Stake, R.

1999 Investigación con Estudios de Casos. Morata, Madrid.

\section{Starkey, L}

2010 Teachers' pedagogical reasoning and action in the digital age. Teachers and Teaching: Theory and Practice 16(2):233-244. doi:10.1080/13540600903478433

\section{Notas}

1 Este cuadro resumen ha sido un producto de la tesis doctoral "Concepciones del profesor sobre aprender y enseñar Historia y su relación con tipos de usos educativos de las TIC". Universitat Oberta de Cataluyna, octubre 2012. Véase el documento completo en: http://www.tdx.cat/ handle/10803/126403

2 En la columna Nivel Uso de TIC del cuadro 2 el autodiagnóstico que se realizaba a cada profesor mediante un ítem en la entrevista considera los siguientes niveles: Básico (maneja software de productividad de manera instrumental, como por ejemplo elaborar documentos en Word, presentaciones o lista de notas en Excel, navega esporádicamente en internet). Intermedio (a lo anterior se agrega que prepara materiales con uso de software e internet de apoyo a su docencia). Avanzado (lo anterior pero además se comunica e interactúa por la red y sistemas informáticos con autonomía). Experto (todo lo anterior y además es capaz de enseñar a otros/as el uso de herramientas de productividad y apoyar a colegas en usos didácticos de las TIC). 
